\title{
Energy Efficient Topology Control Algorithm for Wireless Mesh Networks
}

\author{
${ }^{\mathrm{a}}$ F. O. Aron*, ${ }^{\mathrm{b}}$ T. O. Olwal, ${ }^{\mathrm{a}}$ A. Kurien and ${ }^{\mathrm{c}} \mathrm{M}$. O. Odhiambo. \\ ${ }^{a}$ Dept. of Electrical Engineering, F'SATIE, Tshwane University of Technology \\ ${ }^{\mathrm{b}}$ The Meraka Institute, Council of Scientific and Industrial Research \\ ${ }^{\mathrm{c}}$ Dept. of Electrical Engineering, University of South Africa

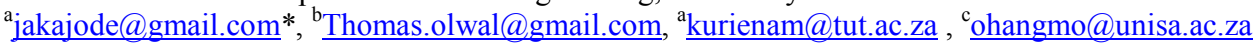

\begin{abstract}
The control of the topology of a network makes it possible for the network nodes to reduce their power of transmission while ensuring that network connectivity is preserved. This paper explains the need for energy consumption control in Wireless Mesh Networks (WMNs) and proposes a Local Minimum Shortest-Path Tree (LM-SPT) algorithm for topology control for the WMNs. The algorithm is distributed with each node using only the information gathered locally to determine its own transmission power. The implementation is done in two phases. The construction of a minimum local shortest-path tree is first done. The removal of all unidirectional links is then done. The performance of the algorithm is demonstrated via several simulation tests. The resultant network topology preserves network connectivity in addition to possessing other desirable features such as: (1) reduction in the average node degree, (2) evenly distributed power consumption among the nodes as well as (3) a reduced total power consumption leading to longer connectivity periods.
\end{abstract}

Keywords - Topology Control, WMNs, Energy Efficiency, Localized Algorithm

\section{INTRODUCTION}

In Wireless Mesh Networks (WMNs), each node operates both as a host and a router. The nodes in the network automatically establish an Ad Hoc network and maintain mesh connectivity. The nodes dynamically self organize and self configure and can be viewed as special cases of Ad Hoc networks. Communication between the nodes is in a multihop fashion. The general architecture of WMNs described by the authors in [1][2][3] is composed of three distinct wireless network elements: a Network Gateway (a mesh router with gateway/bridge functionalities), Access Points (mesh routers) and mobile or stationary nodes (mesh clients). For the purpose of this work, the elements are referred to as mesh nodes (MNs). Real-world WMNs applications [4] have been witnessed in metropolitan area networking, broadband home networking, community and neighbourhood networks, enterprise networking and building automation.

Energy efficiency [5] has been a major topic of discussion in the history of Multihop Wireless Networks (MWNs), such as MANETs, for quite a long time. Issues of concern include the possibility of efficiently using per node energy with a view to lengthening the lifetime of the said network. Other benefits of controlling the energy usage include, the increase in capacity due to the spatial reuse [6] and reduction of node to node interferences during communication.

This work was supported in part by the Tshwane University of Technology, the French South African Institute in Electronics and the University of South Africa.
Although several contributions have been tailored towards studying power control problems in energy-constrained conventional IEEE 802.11 wireless network standards, little attention has been drawn to the power control problems in WMNs. This is mainly because the backbone wireless mesh routers are static and have usually been assumed [4] to have electrical mains power supply, and hence, are purported not to have power constraints. However, with specific considerations to rural area applications of WMNs, we argue that the mesh routers would be stationary, but with power constraints. In rural areas, electrical mains power sources are limited and/or often not available. The mesh nodes have thus to rely on exhaustible and renewable means of energy supply such as solar, battery or generator. Furthermore, the mesh clients are definitely power constrained [4]. In order to address these constraints, the LM-SPT is presented which is a distributed localized energy efficient topology control algorithm for WMNs in rural areas. The algorithm computes the best path based on the link weight functions. The algorithm has similar objectives to [7][8][9][10] but more inclined to WMNs.

The remainder of this paper is organized as follows. In section 2 , the details of the network model is given. In section 3 , previous work related mostly to other MWNs is reviewed. The proposed algorithm is presented in section 4 . Section 5 gives the simulation results and analysis. Finally, in section 6 the work is concluded and a suggestion for future work is given.

\section{NETWORK MODEL}

Consider a set $V=\left\{v_{1}, v_{2}, \ldots \ldots, v_{n}\right\}$ of randomly distributed static MNs located on a 2D plane, each node $u \in V$ has a unique $i d\left(u_{i}\right)=i$, where $1 \leq i \leq N(N=|V|$, number of nodes) and is specified by its coordinates $(x(u), y(u))$ at any instance. Each node $u \in V$ is assigned a power function $p_{t x}$ where $p_{t x}\left(d_{u}\right)$ is the minimum transmission power needed to establish a communication link to another node $v \in V$ located $d_{u}$ distance away from $u$. The maximum transmission power for every node is assumed to be the same and is given by $p_{t x}^{\max }$. The maximum distance needed for any two nodes $u, v \in V$ to communicate directly is also assumed to be the same and is given by $D$. Therefore, $p_{t x}(D)=p_{t x}^{\max }, \forall u \in V$. It is assumed that, there exists an underlying MAC layer that 
helps resolve any interference problems caused by multiple simultaneous transmissions. At the beginning of simulation, every node transmits with full power $p_{t x}^{\max }$ and an induced graph modelled as a quasi Unit Disk Graph (qUDG) $G=(V, E)$ is created. Here, $V$ is the set of all nodes in the network and $E$ is the set of all links/edges i.e., $E=\{(u, v) \mid d(u, v) \leq D\}$.

The WMN topology is modelled as a weight directed graph in which for each edge $(u, v) \in E$, node $v$ has to be within the transmission range of $u$. The notation $d(u, v)$ denotes the Euclidean distance between the nodes $u$ and $v$.

The following gives a list of definitions to the terms that will be used in the paper.

Definition 1 (Accessible Neighbourhood Set): The Accessible Neighbourhood Set, $A_{u}^{N}$, is defined as the set of all nodes that have a direct link with node $u$, when $u$ transmits at maximum transmission power. The set is given by $A_{u}^{N}=\{v \in V \mid d(u, v) \leq D\}$.

Definition 2 (Weight Function): An edge $(u, v)$ has a weight given by the following expression:

$$
w(u, v)=t . d(u, v)^{\alpha}+r x(u, v),
$$

where $t$ is a threshold related to signal to noise ratio at node $u$ and $\alpha \in[2,5]$ is a constant real number depending on the wireless transmission environment. Both parts of (1) are summed up to give the transmission power. The first part is the transmitter power consumed by transmitting a packet from node $u$ to $v$ and $r x(u, v)$ is the receiver power. Assuming all receivers have the same threshold power for signal detection hence the value of $t$ becomes some appropriate constant.

Definition 3 (Logical Neighbour Set): A logical neighbour set of node $u$ is given by $N S_{u}^{L}$. Node $v \in N S_{u}^{L}$ if and only if there exists an edge $(u, v)$ in the topology generated by the algorithm and $N S_{u}^{L}=\{v \in V \mid u \rightarrow v\}$.

Definition 4 (Fully Connected): A network is fully connected if and only if $\forall u \in V$, there exist either a direct path or a multihop path from $u$ to every other node $v \in V$ in the network.

Definition 5 (Relay Region): Given a node $v$, let the physical location of $v$ be denoted by $\operatorname{Loc}(v)$. The relay region of the transmit-relay node pair $(u, v)$ is the physical region $R L_{u \rightarrow v}$ such that relaying through $v$ to any other point in $R L_{u \rightarrow v}$ consumes a lesser power than direct transmission to that point.

Definition 6 (Network Lifetime): Given a set of nodes $V$ and for all $v \in V$ an energy value $E(v)$, the lifetime of node $v$ is $L t_{v}=\left\{t \mid f_{v}(t) \leq E(v)\right\}$ until when $E(v)=0$, where $f_{v}(t)$ is the energy consumed by $v$. The network lifetime
$L t_{V}=\operatorname{Min}_{v \in V}\left(L t_{v}\right)$ i.e., the time taken till the first node goes off.

Definition 7 (Bi-directionality): A topology $G^{\prime \prime}=\left(V^{\prime \prime}, E^{\prime \prime}\right)$ generated by the algorithm is bi-directional if $V^{\prime \prime}=V$, $E^{\prime \prime}=\left\{(u, v) \mid(u, v) \in E\left(G^{\prime}\right)\right.$ and $\left.(v, u) \in E\left(G^{\prime}\right)\right\}$

It is assumed that the qUDG is fully connected. Each node uses an omni-directional antenna to transmit and receive signals. It is also assumed that each node is able to individually adjust its own transmission power. It is further assumed that the wireless channel is symmetric and each node has a GPS for outdoor applications and pseudolite for indoor applications [11] and each node is capable of gathering its location information.

The objective of the topology control algorithm is to find a subgraph of the qUDG, $G=(V, E)$, such that the resultant topology satisfies certain requirements namely, decrease in average node degree, an averagely low power consumption thus longer network lifetime and a maintenance of connectivity in the resultant network topology. Each node must adjust its transmission radius to reduce its power consumption while still maintaining the connectivity.

The algorithm assumes a hybrid WMN [4] which is infrastructure-less and hence has to be distributed and constructed in a localized manner to avoid flooding of the network, i.e., a node must decide its transmission power based only on the information of the nodes reachable by a small constant average number of hops.

\section{RELATED WORK}

Rodoplu and Meng [12] describe the first algorithm which is based on the concept of relay region. A node decides to relay through other nodes if less power will be consumed. The algorithm guarantees the preservation of minimum energy paths between every pair of nodes connected in the original graph. Based on the results of [12], Li and Halpern [13] proposed an improved protocol which is computationally simpler and better in performance with the resulting topology being a sub-network of the one generated by [12]. Li and Halpern [14] further propose the small minimum energy communication network (SMECN). In this algorithm, each node $u$ initially broadcasts "hello" message with some initial power and after reception of $A C K s$ from the receiving nodes, checks if the current range covers the region of maximal transmission range less the union of the compliment of the relay regions of all the nodes reachable by node $u$. The process terminates if node $u$ reaches its maximal transmission power. The work in [12][13] and [14], however, implicitly assume that a long link consumes more power than a shorter link, an assumption that is not practical for instance in heterogeneous networks according to [6].

In $[8][9][10]$, the concept of local neighbourhood is first introduced. This concept proposes that a logical topological view of a node in a network be constructed based only on its local information. This forms the basis of the family of the distributed and localized topology control algorithms. In the 
work of Li et al [8], a node builds its local minimum spanning tree (LMST) based only on its one hop neighbourhood information. It keeps only the one hop nodes as neighbours in the final topology. The resulting topology has been shown to be connected and with node degree bounded by six. In addition they provide an optional phase where the topology is transformed to one with bidirectional links only. The work in [8] however, does not guarantee a maintenance of the minimum energy paths between any two nodes. Another angle of approach is given by $\mathrm{Li}$ et al [7] for heterogeneous networks, in which the resulting network contains unidirectional links.

Other old variants of topology control algorithms such as in [15] also discuss a distributed and localized algorithm to obtain a reliable high throughput topology by adjusting a per node transmission power. However, their focus is not on minimizing the energy consumed in the network.

All of the algorithms shown in [8][7][9][10][15] have not been applied to WMNs. It can not be generally assumed that the algorithms will automatically function in WMNs since the requirements on power efficiency and mobility are very different between WMNs and other MWNs [4]. The algorithm proposed in this work consists of two phases with the resulting topology ensuring connectivity and reduced node logical out degrees and is shown to apply for WMNs.

\section{PROPOSED ALGORITHM}

A two phased design algorithm i.e., construction of a Local Minimum Shortest-path Tree and unidirectional links removal is proposed in this paper.

\section{Phase 1: Construction of Local Minimum Shortest-path Tree (LM-SPT).}

In this phase, each node gathers neighbour information and constructs an LM-SPT (as depicted in Figure 2). The phase involves three stages:

a) Information collection and exchange:- Each node in this step periodically broadcasts a beacon 'Hello' messages using maximum power $p_{t x}^{\max }$. The information exchanged here includes the node ID and the position in the 2D plane. This information is used to calculate the node to node distance, the link weights and the path weights. The link weight represents the power required for transmission along a link, and the path weight represents the sum of all minimum link weights of a path from source to destination. The result of this stage is the 'Accessible Neighbourhood Set' $A_{u}^{N}$ for each node $u \in V$. The 'Hello' message is also sent by each node asynchronously and periodically giving each node's information about its neighbours.

b) Construction of a Logical Visible Neighbourhood Topology: - Each node applies the concept of the relay region in order to gather the nodes in the set of 'Logical Visible Neighbourhood'. The set $\operatorname{LVN}(u, k) \subseteq A_{u}^{N}$ at $k=1$. If a node $v \in A_{u}^{N}$ is in the relay region of another node $w \in A_{u}^{N}$ then node $v$ is moved to a new set of non neighbours called NotNbr. This is repeated for all the nodes $i \in A_{u}^{N}$. All nodes reachable via other nodes are moved out of the set $A_{u}^{N}$ and the remaining set is called $L V N(u)$. Each node then applies the Dijkstra's algorithm independently from a source node to all the other nodes in $V$ in order to build its LM-SPT.

c) Computing the transmission power: - Each node computes its minimal transmission power to cover only all of the nodes contained in the set $\operatorname{LVN}(u, k)$, in which case, it determines which node among the nodes is furthest. The node then adjusts its transmission power to reach this node and thus all other nodes in the set $\operatorname{LVN}(u, k)$ are covered. The set is given by $G^{\prime}=\left(V^{\prime}, E^{\prime}\right)$. From the information on the location of the nodes, the inter node distance is calculated. The distance is applied in the propagation model formulas to obtain the minimum transmission power. The free space model is used for short distances and the two ray ground reflection model is used for longer distances depending on the value of the Euclidean distance in relation to the cross over distance.

The cross over distance is calculated using the following expression:

$$
\text { Cross_Over_dist }=\frac{4 \pi h_{t} h_{r}}{\lambda},
$$

where $h_{t}=h_{r}=1.5$, are the antenna heights of the transmitter and receiver respectively. Lambda, $\lambda$, denotes the wavelength. For $d(u, v)<$ Cross_over_dist, the Free Space model is used. Whereas For $d(u, v) \geq$ Cross_over_dist, the Two-ray-ground model is used. The free-space propagation model is given by the following expression:

$$
P \min =\frac{R x \operatorname{Thresh}\left((4 \pi d)^{2} L\right)}{G_{t} G_{r} \lambda^{2}} .
$$

The two ray ground reflection model is given by the following expression:

$$
\operatorname{Pmin}=\frac{R x \operatorname{Thresh}\left(d^{4} L\right)}{G_{t} G_{r} h_{t}^{2} h_{r}^{2}},
$$

where $G_{t}=G_{r}=1$ is the transmitter and receiver Antenna gain respectively, $L=1$ is path loss exponent and again the values of $h_{t}=h_{r}=1.5$.

Figure 1 shows the algorithm that runs in each node $u \in V$ to compute the minimum transmission power. Let $p(u, v)$ be the minimum power required to transmit a data packet from node $u$ to node $v$ at any time instance. Also let initial power $p=p_{t x}^{\max }$ and $F(u, p)$ be the region that node $u$ can reach if it broadcasts with power $p$. It is assumed that every node $u$ 
knows its terrain and antennae characteristics and is able to compute the region $F(u, p)$.

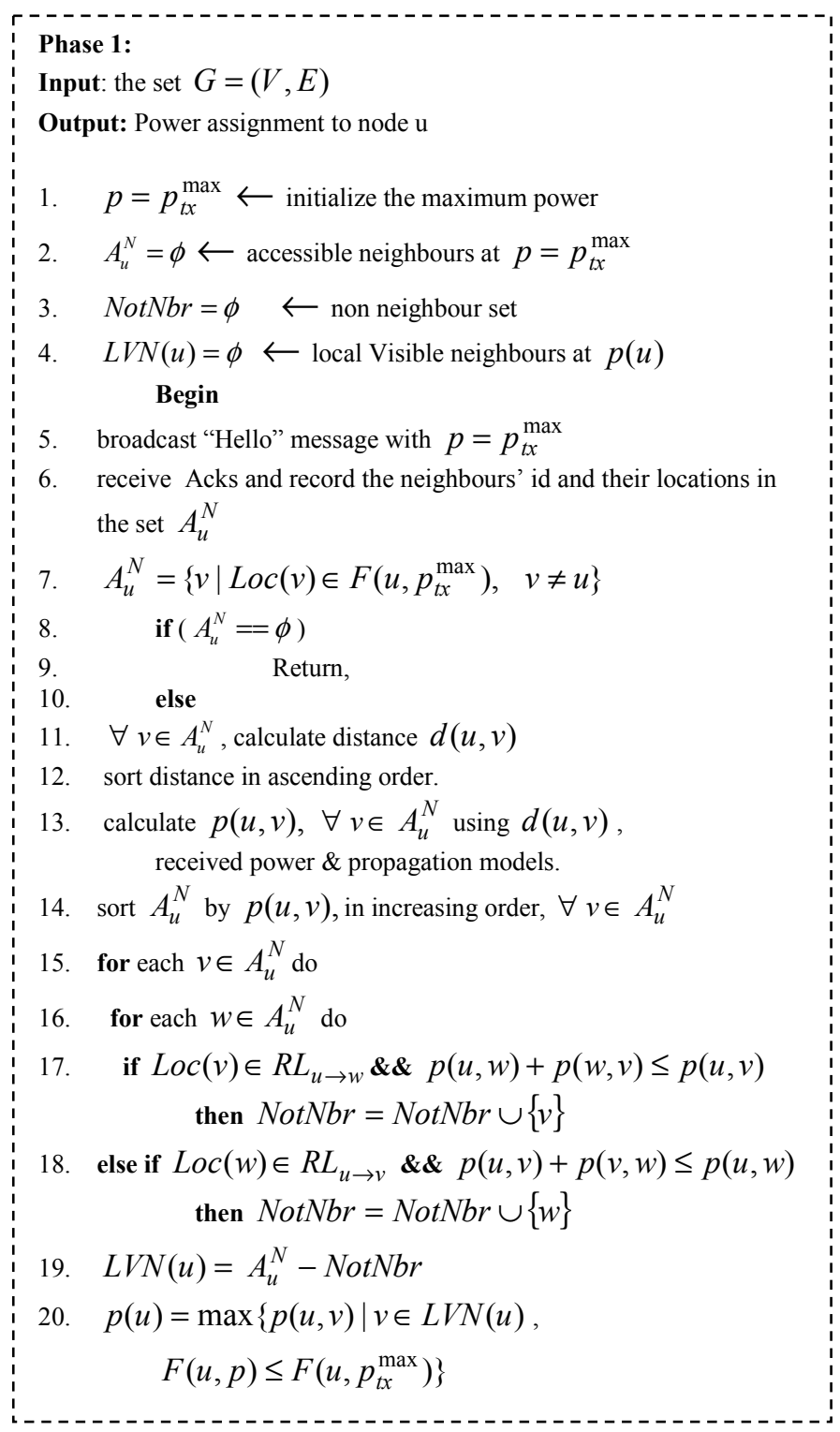

Figure 1: Algorithm used to calculate the transmit power at node u.

The sets Accessible Neighbourhood $\left(A_{u}^{N}\right)$, Not neighbour ( NotNbr), and Logical Visible Neighbourhood ( $L V N(u)$ ) of node $u$ are initialized to empty set, $\phi$. Node $u$ broadcasts the 'Hello' messages at full transmission power $p_{t x}^{\max }$ stating its position. It collects all the $A C K s$ recording each nodes ID, and Location $(\operatorname{Loc}(v))$ in the Accessible Neighbourhood set $A_{u}^{N}=\left\{v \mid \operatorname{Loc}(v) \in F\left(u, p_{t x}^{\max }\right), \quad v \neq u\right\}$ where $\operatorname{Loc}(v)$ is the location of the node $v$ and $F\left(u, p_{t x}^{\max }\right)$ is the region covered by node $u$ at full transmission power.

For every node $v \in A_{u}^{N}$, node $u$ computes the distance $d(u, v)$ and the power $p(u, v)$ and arranges them in ascending order. For every two nodes $v, w \in A_{u}^{N}$, if node $v$ is in the relay region of node $w$ i.e., $\operatorname{Loc}(v) \in R L_{u \rightarrow w}$ and $p(u, w)+p(w, v) \leq p(u, v)$ then node $v$ is moved to the NotNbr set, otherwise it remains. If $\operatorname{Loc}(w) \in R L_{u \rightarrow v}$ and $p(u, v)+p(v, w) \leq p(u, w)$ then node $w$ is moved to the NotNbr set otherwise it remains. The Logical Visible Neighbourhood of node $u$ i.e., $\operatorname{LVN}(u)$ is therefore given by the set $A_{u}^{N}$ less NotNbr set of node $u$.

\section{Phase 2: Removal of Unidirectional links.}

Bi-directional links are quite important for link level acknowledgements and for packet transmissions and retransmissions over the unreliable wireless medium. In phase two of the algorithm, unidirectional links generated in phase 1 are removed so as to obtain bi-directional edges using edge addition. The resulting topology is given by $G^{\prime \prime}=\left(V^{\prime \prime}, E^{\prime \prime}\right)$ where $V^{\prime \prime}=V, E^{\prime \prime}=\left\{(u, v) \mid(u, v) \in E\left(G^{\prime}\right)\right.$ and $\left.(v, u) \in E\left(G^{\prime}\right)\right\}$. Figure 2 depicts the algorithm used. For every node $v \in L V N(u)$, if there is an edge $u \rightarrow v$, then there must be an edge $v \rightarrow u$ and node $u \in \operatorname{LVN}(v)$.

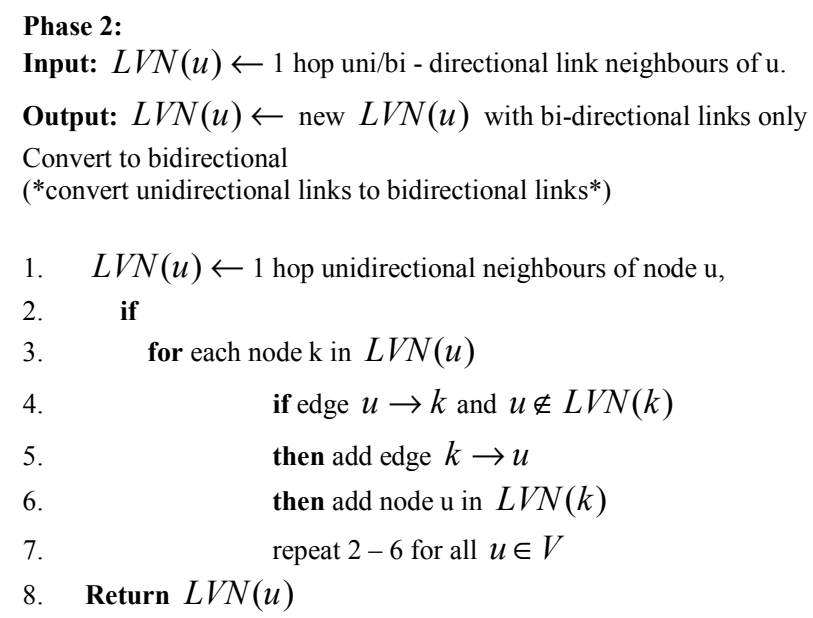

Figure 2: Algorithm used for removal of unidirectional

Theorem 1: the resultant network topology $G^{\prime \prime}$ ensures that if $G$ is fully connected, then $G^{\prime \prime}$ is also fully connected.

Proof: taking arbitrarily any two nodes $u, v \in V$ and based on the previous assumption that the qUDG is fully connected and by definition 4 , there exist a bidirectional path from node $u$ to $v$ denoted by $u \leftrightarrow v$. We prove that there is a path from $u$ to $v$ in $G^{\prime \prime}$.

In stage 1 of phase 1 of the scheme, the local node $u$ collects all the direct links to nodes in $A_{u}^{N}$. For all nodes $v \in V$, if $v \in A_{u}^{N}$, then $u \leftrightarrow v$. In stage 2 , based on the edge weights, node $u$ constructs the minimum shortest path tree using the concept of Relay region and the Dijkstra's shortest path algorithm. Under the Relay region concept, if $w, k \in A_{u}^{N}$ and $\operatorname{Loc}(v) \in R L_{u \rightarrow w}$ and $p(u, w)+p(w, v) \leq p(u, v)$ then link 
$u \leftrightarrow v$ is deleted. This implies that $u \leftrightarrow v$ becomes $u \leftrightarrow w$, $w \leftrightarrow v$. Otherwise, if $\operatorname{Loc}(w) \in R L_{u \rightarrow v}$ and $p(u, v)+p(v, w)$ $\leq p(u, w)$ then link $u \leftrightarrow w$ is deleted, and $u \leftrightarrow w$ becomes $u \leftrightarrow v, v \leftrightarrow w$. This implies no path is lost. The Dijkstra's shortest path algorithm includes all the nodes derived in the $L V N(u)$ and only constructs a local shortest path tree. In stage 3 , the required transmission power per node is determined based on the longest edge in $\operatorname{LVN}(u)$ guaranteeing that all nodes in the set are covered. Finally, in phase 2, bidirectional links are constructed by link addition which does not lead to loss of the already constructed links. From the 2 phases, it is clear that even though the direct edge from $u \leftrightarrow v$ may be deleted after the second stage because of the availability of a more power efficient path, a path from $u$ to $v$ exists and is guaranteed to be valid.

\section{SIMULATION AND RESULTS}

In this section, some of the simulation results to verify the effectiveness of LM-SPT are presented. The performance of LM-SPT is compared to that of LMST [8] and the IEEE 802.11b Maximum Power. The topology control algorithm is implemented in NS-2. The nodes are randomly distributed in a rectangular region of $670 \mathrm{~m} \times 670 \mathrm{~m}$ and are varied in number from 10 to 100 nodes. All the nodes have a maximum transmission range $D$ of $250 \mathrm{~m}$. A carrier frequency of $2.4 \mathrm{GHz}$ and a transmission bandwidth of $2 \mathrm{MHz}$ is used. It is assumed that the omni-directional antennas used have a $0 \mathrm{~dB}$ gain and are placed at a height of $1.5 \mathrm{~m}$ above a node. The OLSR is used as the routing protocol in the simulations due to its distributive nature. UDP traffic is used as the application traffic source with the number of connections varying from 10 , 20,30 or 40 .

The average connectivity is obtained by evaluating the average node degree (the mean connectivity per node) using the formula $C_{u}=y /(N)$, where $y$ is the number of nodes reachable by node $u$ and $N$ is the total number of nodes in the network. The average mean connectivity denoted by $\psi$ is given by:

$$
\psi=\frac{1}{N} \sum_{u=0}^{N-1} C_{u},
$$

which is equivalent to summing up all the mean connectivity of every node in the entire network. The value of $C_{u}$ should not be too large as this would imply that a node communicates even with very distant nodes and this increases interference and collision and also wastes energy. On the other hand, it should not be made too small as this would imply that longer paths have to be taken to reach destinations and this also increases the overall energy consumption in the network. Figure 3 shows a comparison of the average node degree levels. The LM-SPT algorithm records a great reduction in the average node degree as compared to MaxPower. It has a performance close to LMST. Even though LMST outperforms LM-SPT in terms of node degree, LMST does not preserve the minimum energy paths. Hence, shorter network lifetimes as shown in figure 5 and 6.

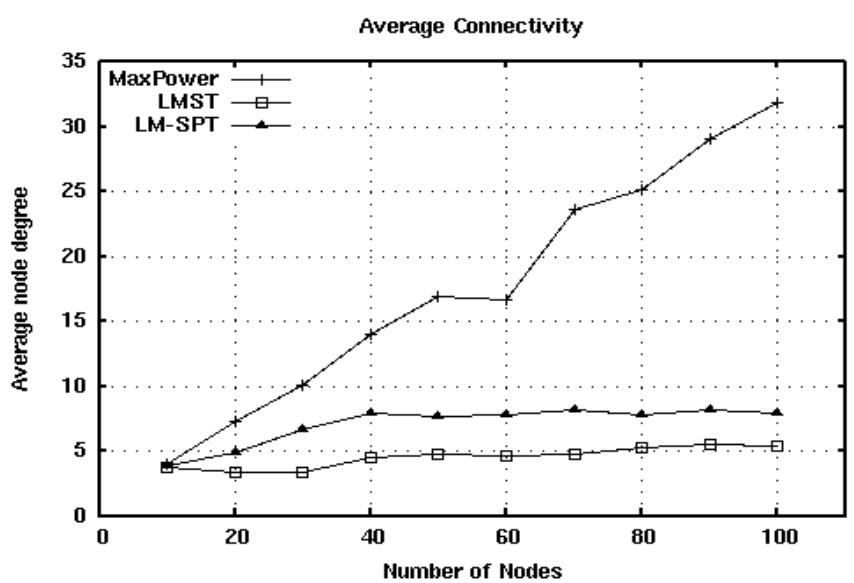

Figure 3: Performance comparison in terms of Average Node Degree. Nodes range from 10 to 100 .

The lifetime of each of the network instances is considered. This is measured in terms of the number of nodes that remain alive over a period of time. The simulations are based on the assumption that nodes are static and are run for a period of 300 seconds. Figure 4 shows the lifetime of a network of 50 nodes with 20 traffic connections at random times.

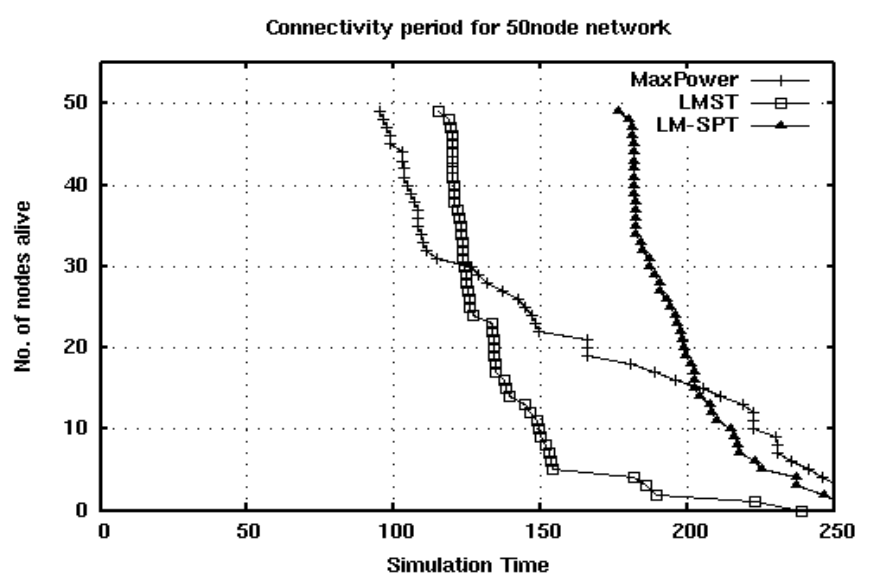

Figure 4: Performance comparisons in terms of network lifetime for a 50nodes network.

A total of 1024 packets were sent with 512 bytes of data. It is noted that when using maximum transmission power, the network lifetime ends after around 98 seconds. LM-SPT performs better than MaxPower and LMST. At reduced optimal per node transmission energy, channel contention is reduced and a nodes total amount of processing power is reduced as it only reaches few neighbours and eventually the overall consumed power in the network is reduced. With too few neighbours, as shown by LMST, there may occur several shorter paths in a single path to a destination leading to more power consumed per path and hence less lifetime.

Similarly, in figure 5, a network of 100 nodes is simulated with 40 traffic connections at random times. Again LM-SPT is shown to be superior, extending the network lifetime to over 120 s as compared to 103 s of MaxPower and 110s of LMST. 


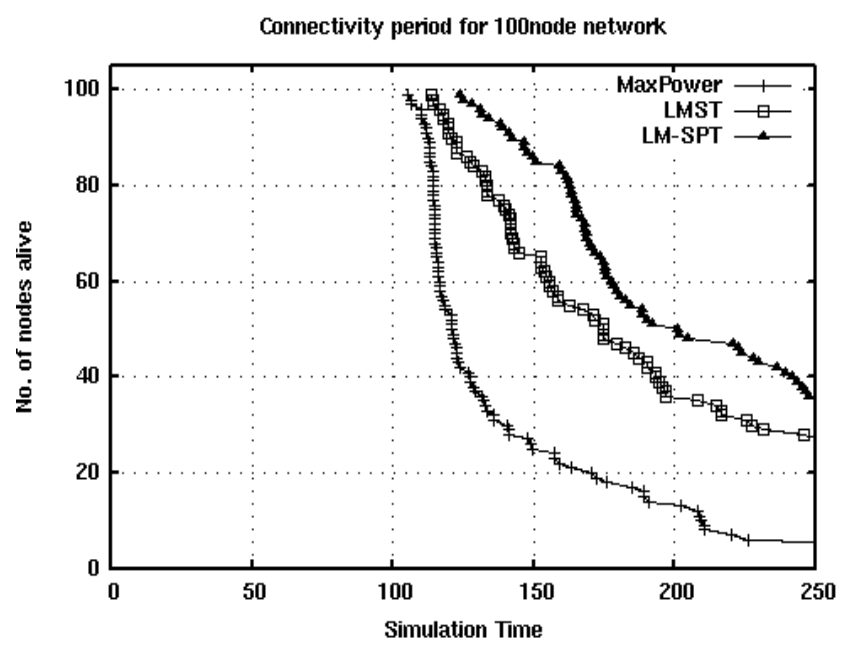

Figure 5: Performance comparisons in terms of network lifetime for a 100 nodes network.

Additionally, the time complexity of the proposed scheme is discussed phase by phase. Let $N_{i}$ and $E_{i}$ denote the number of nodes and edges respectively in the $i^{\text {th }}-$ hop neighbourhood. From section 2, $N=|V|$. The cost of Dijkstra's algorithm is known to be $O(N \log N+E)$ when priority queue is implemented in a Fibonacci heap. Therefore, in phase 1 of our scheme, each node requires $O\left(N_{1} \log N_{1}+E_{1}\right)$ to complete. This results to a time complexity of $O\left(N^{2}\right)$ in the worst case. Similarly, in phase 2, each node executes the search for unidirectional links once. This results in a computational cost of $O\left(N_{k} \log N_{k}+E_{k}\right)$ which is also $O\left(N^{2}\right)$ in the worst case. Hence, the time complexity for the whole execution of the algorithm is $O\left(N^{2}\right)$.

\section{CONLUSION}

The lifetime of multihop wireless networks such as WMNs whose mesh nodes have limited or no mains power supply and rely on exhaustible and renewable means of energy supply can be greatly increased by the efficient management of power consumption in each individual node in the network. In order for the effectiveness of these networks to be realized, the lifetime of the network is very critical. In this work, an enhanced minimum shortest-path tree based energy efficient topology control algorithm (LM-SPT) for wireless mesh networks with limited mobility was presented. The algorithm uses only the locally available information to determine the nodes that should be its logical neighbours at any given time. The resulting topology ensures connectivity, lower average node degree as well as reduced power consumption in the network. The algorithm was validated via simulations on the ns-2 platform. The work was based on the assumption of stationary nodes. Future work will extend the algorithm to consider a more mobile environment at the mesh clients' side where node mobility is highly considered and to extend the algorithm to cover for a hybrid heterogeneous mesh network.

\section{REFERENCES}

[1] S. Waharte, R. Boutaba, Y. Iraqi and B. Ishibashi, "Routing protocols in wireless mesh networks: challenges and design considerations," to appear in the Multimedia Tools and Applications (MTAP) Journal, Special Issue on Advances in Consumer Communications and Networking, 2005.

[2] S. Waharte and R. Boutaba, "Tree-based WirelessMesh Network Architecture," In Proceedings. $1^{\text {st }}$ Intnl. Workshop on Wireless Mesh Networks (MeshNets), Budapest, Hungary, July 2005.

[3] Nortel Networks Wireless Mesh Networks Solution. http://www.nortelnetworks.com/solutions/wrlsmesh/ architecture.html.

[4] I. F. Akyildiz, X. Wang and W. Wang, "Wireless mesh networks: a survey," In Computer Networks, vol. 47, pp. 445-487, 2005.

[5] C.E. Jones, K. M. Sivalingam, P. Argrawal, and J.C. Chen, "A survey of energy efficient network topologies for wireless networks," wireless networks, vol 7, no. 4, pp. 343-358, Aug 2001.

[6] Yao Shen, Yunze Cai, Xiaoming Xu, "A shortest-path-based topology control algorithm in wireless multihop networks". Computer Communication Review 37(5), pp. 29-38, 2007

[7] N. Li and J. Hou, "Topology Control in Heterogeneous Wireless Networks: Problems and Solutions", IEEE Infocom, 2004

[8] N. Li, J. Hou, L. Sha, "Design and analysis of an MST-based distributed topology control algorithm", in: Proceedings IEEE INFOCOM, June 2003.

[9] S.C. Wang, D.S.L. Wei, S.Y. Kuo, "A topology control algorithm for constructing power efficient wireless ad hoc networks", in: Proceedings IEEE GLOBECOM, December 2003.

[10] X.-Y. Li, "Approximate MST for UDG locally", in: Proceedings International Computing and Combinatorics Conference (COCOON), July 2003.

[11] T. He, C. Huang, B. M. Blum, J. A. Stankovic, and T. Abdelzaher, "Range-free localization schemes for large scale sensor networks," in Proceedings. ACM MOBICOM 2003, San Diego, California, United States, Sept. 2003, pp. 81-95.

[12] V. Rodoplu and T.H. Meng, "Minimum energy mobile wireless networks". IEEE Journal on Selected Areas in Communications, 17(8):1333-1344, 1999.

[13] L. Li, J. Halpern, "Minimum energy mobile wireless networks revised", in: Proceedings IEEE ICC, June 2001.

[14] L. Li and J.Y. Halpern, "A minimum-energy path-preserving topologycontrol algorithm" IEEE Transactions on Wireless Communications, 3(3):910-921, 2004.

[15] L. Hu, "Topology Control for Multihop Packet radio Networks", IEEE Transactions on Communications, 41(10), pp 1474-1481, 1993.

[16] A.E.F. Clementi, P. Penna, R. Silvestri, "On the power assignment problem in radio networks," in: Electronic Colloquium on Computational Complexity (ECCC), 2000.

[17] N. Li and J. C. Hou, "Localized topology control algorithms for heterogeneous wireless networks", IEEE/ACM Transactions on Networking, 13(6):1313-1324, 2005. 